Original Article

\title{
Trophoblast type-specific expression of senescence markers in the human placenta
}

\author{
Shotaro Higuchi, Tsutomu Miyamoto*, Hisanori Kobara, Satoshi Yamada, Ryoichi Asaka, \\ Norihiko Kikuchi, Hiroyasu Kashima, Satoshi Ohira, Tanri Shiozawa
}

Department of Obstetrics and Gynecology, Shinshu University School of Medicine, 3-1-1 Asahi, Matsumoto, 390-8621, Japan

\section{A R T I C L E I N F O}

\section{Keywords:}

Cytotrophoblast

Syncytiotrophoblast

Cell senescence

Placenta

Senescence-associated beta-galactosidase

\begin{abstract}
A B S T R A C T
Objectives: Cell senescence is irreversible cell cycle arrest. The human placenta is a unique organ that grows and matures during pregnancy until 40 weeks of gestation. However, the role of senescence in placental villi, particularly in the two types of trophoblast, has not yet been elucidated in detail. Therefore, we herein investigated the expression of cell senescence-related markers in trophoblast.

Methods: Seventy normal placental tissues were used. The expression of senescence-associated beta-galactosidase (SA- $\beta$-gal), cell senescence-related markers (p16, p21, and promyelocytic leukemia; PML), and a growth marker (MCM2) was immunohistochemically examined. The expression of these markers in BeWo cells before and after cell fusion using forskolin was also investigated.

Results: The expression of MCM2 is detected in cytotrophoblast (CT). The expression of SA- $\beta$-gal in CT is strong in the first and second trimesters, but weaker in the third trimester. Syncytiotrophoblast (ST) are negative in the first and second trimesters, but become positive in the third trimester. The immunohistochemical expression of p16, p21, and PML is stronger in CT than in ST throughout pregnancy. Furthermore, the expression of these markers in ST significantly increases as pregnancy advances. The expression of SA- $\beta$-gal, PML, and p21 in BeWo cells is stronger after than before cell fusion.

Conclusions: The proliferation and senescence of CT occurred in early to mid-pregnancy in association with syncytial fusion, while senescence was observed in ST in late pregnancy. This coordinated trophoblastic senescence may be essential for maintaining placental function.
\end{abstract}

\section{Introduction}

The uterus is maintained in a static condition from implantation until the beginning of parturition at 40 weeks of gestation. The mechanisms underlying parturition in humans have not yet been elucidated in detail because of its complexity. Many factors are presumed to be responsible for parturition, including functional progesterone withdrawal [1], the increased production of and sensitivity to oxytocin and prostaglandin [2], fetal corticotrophin-releasing hormone [3], and inflammatory cytokines [4]. Of these, functional progesterone withdrawal and inflammatory cytokine theories have recently been examined in detail because the mechanisms responsible for the onset of preterm labor are considered to be similar to those of term labor, and treatments with progesterone and antibiotics have demonstrated effectiveness against threatened premature labor. However, the human "clock" mechanism that induces labor 280 days after conception currently remains unclear.
Inflammatory cytokines play important roles in the induction of parturition. One of the critical triggers for spontaneous preterm birth is bacterial infection [5]. Bacterial vaginosis and cervicitis induce leukocyte migration and phagocytosis. Consequently, an increase in cytokines leads to cervical ripening, membrane rupture, and parturition. In labor, the levels of inflammatory cytokines, such as IL-6 and IL-8, are increased in the cervix and uterine muscle, which activate matrix metallopeptidase 9 (MMP-9) and induce cervical ripening [6]. Nevertheless, labor in term is not generally accompanied by infection [7]. In recent years, cell senescence and a senescence-associated secretory phenotype (SASP) have been the focus of research to establish why sterile inflammation occurs $[8,9]$. Senescent cells secrete several inflammatory factors [9]. Therefore, the inflammatory cytokines secreted from senescent cells may be involved in the onset of labor.

The placental villus consists of two trophoblastic cell types: cytotrophoblast (CT) and syncytiotrophoblast (ST) form the inner and outer layers of the villus, respectively [10]. In the first trimester, villi have

\footnotetext{
* Corresponding author.

E-mail address: tmiya@shinshu-u.ac.jp (T. Miyamoto).
} 
two layers of these cell types. As gestational weeks proceed, inner CT are absorbed by outer ST. This phenomenon is referred to as syncytial fusion [11], and may be mediated by cellar senescence because this fusion is considered to indicate the terminal stage of CT [12]. Furthermore, in ST, the onset of labor may correlate with clinical aging because placental maturity has been reported in terms of the morphology and function of villi [13]. However, the molecular basis of cell senescence in trophoblastic cells has not yet been elucidated in detail. Therefore, we herein investigated the expression of cell senescencerelated proteins and senescence-associated- $\beta$-galactosidase (SA- $\beta$-gal) positivity in first, second, and third trimester placentas. We also examined the role of cell senescence during the process of cell fusion using BeWo cells as a CT model.

\section{Materials \& methods}

\subsection{Sample preparation}

Trophoblastic tissue samples ranging between 5 and 40 weeks of gestation obtained by induced abortion or elective cesarean section at Shinshu University Hospital were used in the present study. In SA- $\beta$-gal staining, the fresh villus samples used consisted of five cases each of early (5-10 weeks of gestation), mid- (14-20 weeks), and late (37-40 weeks) pregnancy. In immunohistochemical staining, the formalinfixed, paraffin-embedded villus tissues of 10 cases each of early, mid-, and late pregnancy were used. The present study was approved by the Ethics Committee of the institution, and written informed consent was provided by each patient.

\subsection{Cell culture}

Human chorionic carcinoma BeWo cells (ATCC, Manassas, VA) were cultured in Ham's F12K (Life Technologies, Carlsbad, CA) supplemented with $10 \%$ FBS (Life Technologies) at $37{ }^{\circ} \mathrm{C}$ and $5 \% \mathrm{CO}_{2}$. A total of $0.8 \times 10^{6}$ of BeWo cells were seeded on 60 -mm dishes for Western blotting, $0.05 \times 10^{6}$ of BeWo cells were seeded on chamber slides (SANSYO, Iwaki, Japan) for SA- $\beta$-gal staining, and these cells were then cultured overnight. After the medium was changed, $20 \mu \mathrm{M}$ of forskolin (WAKO, Tokyo, Japan) was added. BeWo cells were collected 0, 24, 48, and $72 \mathrm{~h}$ after the addition of forskolin for Western blotting and the culture supernatant was collected for the measurement of hCG concentrations. Cells on the chamber slides were used for SA- $\beta$-gal staining 24 and $48 \mathrm{~h}$ after the addition of forskolin.

\subsection{SA- $\beta$-gal staining}

\subsubsection{Tissue preparation}

Surgically extirpated villus and placental tissues were washed using PBS to remove blood. After fixation by $10 \%$ formalin for five minutes, samples were washed using PBS. After washing, each tissue was fixed overnight in Holt's gum sucrose solution [14] consisting of $300 \mathrm{~mL}$ of deionized water, $5 \mathrm{~g}$ of powdered acacia, $150 \mathrm{~g}$ of sucrose, $100 \mathrm{~mL}$ of $0.5 \mathrm{M}$ phosphate buffer $\mathrm{pH} 7.2$, and $0.1 \mathrm{~g}$ of thymol. Tissues were then embedded in OCT compound and frozen in liquid nitrogen. Five-micrometer-thick tissue sections were obtained using a cryostat.

\subsubsection{Staining procedure}

Specific staining was performed using a Senescence $\beta$-Galactosidase Staining Kit (Cell Signaling Technology, Danvers, MA). Placental tissue sections and BeWo cells in chamber slides were washed twice by PBS, and fixative solution was added at room temperature for $15 \mathrm{~min}$, followed by washing twice with PBS. After the addition of $\beta$-Galactosidase Staining solution, samples were incubated at $37^{\circ} \mathrm{C}$ overnight under dry conditions without $\mathrm{CO}_{2}$. After the incubation, samples were washed twice with PBS. Tissues sections were stained by hematoxylin, dehydrated, and mounted. BeWo cells were mounted with DAPI using
Vectashield (Vector Laboratories, Burlingame, CA), and staining was observed under an inverted microscope (Olympus, Tokyo, Japan). Senescent cells were stained blue by SA- $\beta$-gal staining. The results of staining were semi-quantitatively scored by the ratio of the stained area as follows: 0 : negative ( $0 \%$ stained), 1 : weak $(0-30 \%), 2$ : medium (30-50\%), and strong ( $>50 \%$ ) (Supplementary Fig. S1).

\subsection{Immunohistochemistry (IHC)}

IHC was performed as previously described [15]. Briefly, indirect immunostaining was performed for p16, p21, and PML (promyelocytic leukemia) as senescence markers and MCM7 (minichromosome maintenance protein 7) as a proliferation marker. Tissue sections were deparaffinized with xylene and rehydrated in a graded alcohol series. Antigen retrieval was achieved by a microwave pretreatment in $0.01 \mathrm{M}$ citrate buffer $\mathrm{pH} 6$ for $30 \mathrm{~min}$. Tissue sections were incubated with specific primary antibodies for p16 (rabbit monoclonal, EPR1473, 1:100 dilution, Abcam, Cambridge, UK), p21 (mouse monoclonal antibody, EA10, 1:100 dilution, Abcam), PML (rabbit polyclonal, 1:250 dilution, Abcam), and MCM7 (mouse monoclonal, DCS-141, 1:50 dilution, Santa Cruz Biotechnology, Dallas, TX) at $4{ }^{\circ} \mathrm{C}$ overnight. In the negative control, sections were treated in the same manner, except for being incubated with buffered solution without the primary antibodies. After washing with PBS, tissue sections were incubated with the secondary anti-mouse or rabbit antibody (Histofine Simple Stain MAX PO, Nichirei Biosciences, Tokyo, Japan) at room temperature for $30 \mathrm{~min}$. The immunocomplex was then visualized by diaminobenzidine. Counterstaining was performed using hematoxylin. Immunostaining for Ecadherin (mouse monoclonal antibody, HECD-1, 1:100 dilution, Abcam) and hCG (mouse monoclonal antibody, 5H4-E2, 1:100 dilution, Abcam) in sequential sections was used to identify CT and ST, respectively. The results of staining were semi-quantitatively evaluated by a positivity index (PI, \%), which was defined as the number of positively stained nuclei among 100 nuclei of CT or ST in consecutive fields. The evaluation was performed at 3 fields per case and repeated by 2 investigators (S.H. and T.M.).

\subsection{Immunofluorescent staining}

To further confirm the expression of p16 and p21 in CT or ST, dual staining using immunofluorescence was performed for p16 and hCG, and p21 (mouse monoclonal antibody, CP74, $5 \mu \mathrm{g} / \mathrm{mL}$, Abcam) and Ecadherin (rabbit polyclonal antibody, 1:30 dilution, Abcam), respectively. An Alexa Fluor 488-conjugated goat anti-rabbit IgG antibody and Alexa Fluor 594-conjugated goat anti-mouse IgG antibody (Thermo Fisher Scientific, Waltham, MA) were used as secondary antibodies according to the manufacturer's instructions. Counter nuclear staining by 4',6-diamidino-2-phenylindole (DAPI) was performed using VECTASHIELD Mounting Medium with DAPI (VECTOR Laboratories, Burlingame, CA). Photographs for each fluorescence were taken by a confocal laser microscope (Zeiss LSM 880 with Airyscan, Carl Zeiss, Oberkochen, Germany) and merged.

\subsection{Western blotting}

Proteins extracted from cultured BeWo cells were subjected to a Western blot analysis, as described previously [16]. In brief, proteintransferred membranes were incubated with the primary antibodies for p16 (1:1000 dilution), p21 (1:100 dilution), PML (1:1000 dilution), Ecadherin (mouse monoclonal antibody, HECD-1, 1:1000 dilution, Abcam), and $\beta$-actin (mouse monoclonal, AC-74, 1:5000, Sigma-Aldrich, St. Louis, MO) at $4{ }^{\circ} \mathrm{C}$ overnight. These membranes were then incubated with peroxidase-conjugated secondary antibodies at room temperature for $30 \mathrm{~min}$. Bound antibodies were visualized using the ECL Western blot detection reagent (Amersham, Piscataway, NJ). Proteins extracted from the HPV-positive, p16-overexpressing cervical 
(a)

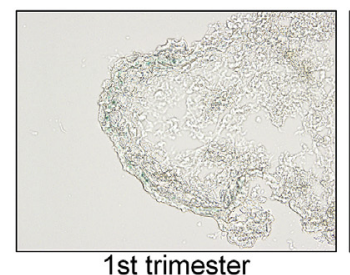

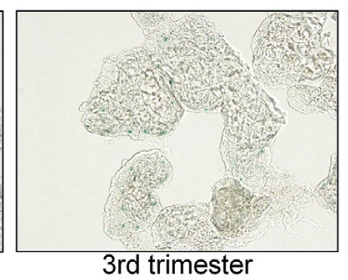

3rd trimester

(b) SA- $\beta$-gal staining score

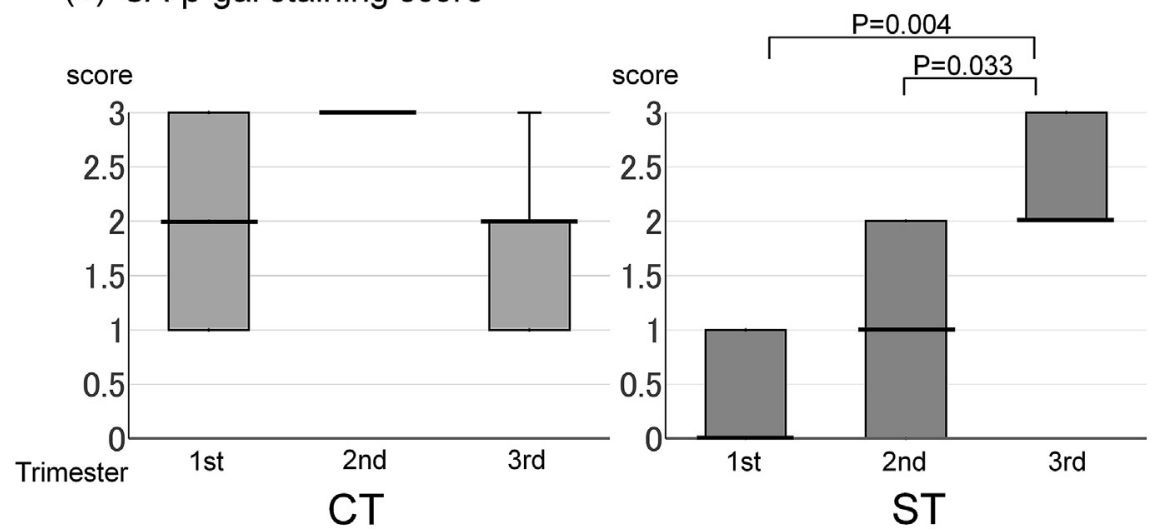

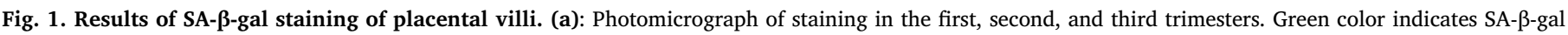
staining. (b): A graphic demonstration of the staining score using a box plot with the median and 25-75 percentile in each trimester.

carcinoma CaSki cell line were used as the positive control for p16. HCG in the culture supernatant was measured by a chemiluminescent enzyme immunoassay in SRL, Inc. (Tokyo, Japan).

\subsection{Statistical analysis}

Statistical analyses were conducted with Scheffé's test.

\section{Results}

\subsection{SA- $\beta$-gal staining}

Positive staining for SA- $\beta$-gal is observed in CT and ST throughout pregnancy (Fig. 1). In CT, the expression of SA- $\beta$-gal is the strongest in the second trimester and decreases in the third trimester; however, this difference is not significant. In ST, the expression of SA- $\beta$-gal is weak in the first trimester, becomes stronger with the progression of pregnancy, and peaks in the third trimester, with its expression being significantly stronger in the third trimester than in the first and second trimesters.

\subsection{Immunostaining for $p 16, p 21$, and $P M L$}

The immunohistochemical expression of the senescence-related proteins, p16, p21, and PML, is observed in CT and ST (Figs. 2 and 3 and Supplementary Figs. S2 and S3). In CT, the PI for p16 in the first, second, and third trimesters are 71, 78, and 73\%, respectively. PI in the first and second trimesters significantly differ $(P=0.043)$. The PI for p21 in the respective three trimesters are 93,93 , and $90 \%$, and those for PML are 67, 73, and 67\%. In ST, the PI for p16 in the first, second, and third trimesters are 14,14 , and $32 \%$, respectively. The PI is significantly higher in the third trimester than in the first $(P=0.011)$ and second $(\mathrm{P}=0.013)$ trimesters. The PI for $\mathrm{p} 21$ in the three trimesters are 24,24 , and $74 \%$, respectively, with that in the third trimester being significantly higher than those in the first $(P=0.001)$ and second $(\mathrm{P}=0.001)$ trimesters. The PI for PML are 6,7 , and $10 \%$, respectively. The expression of p16, p21, and PML is stronger in CT than in ST throughout pregnancy. The expression of these markers in ST increases in a stepwise manner as pregnancy advances. Cell columns containing extravillous trophoblast (EVT) are present in the first trimester. EVT in cell columns also express p16 (Supplementary Fig. S4).

\subsection{Expression of MCM7}

Since the expression of senescence markers in CT increases as early as in the first trimester, we investigate proliferative activity. The expression of MCM7, a proliferation marker, is observed in CT, but is negligible in ST, indicating a similar staining pattern with senescencerelated markers. The PI of MCM7 in CT decreases in the third trimester (85, 82, and $45 \%)$ with a significant difference $(P=0.001, P=0.002$, Figs. $2 \mathrm{~b}, 3 \mathrm{a}$ and $3 \mathrm{~b}$ ), suggesting that $\mathrm{CT}$ grow more vigorously in the first and second trimesters.

\subsection{Fusion of BeWo cells and senescence}

The present results indicate different senescence patterns between CT and ST, and CT are known to be fused into ST. To elucidate the mechanisms underlying cell type-dependent senescence, we focus on the effects of the syncytial fusion of CT on cell senescence. To achieve this, human choriocarcinoma BeWo cells, which reportedly replicate the fusion of CT in vitro, are used. The expression of SA- $\beta$-gal in BeWo cells is enhanced with the addition of forskolin, which induces cell fusion through the up-regulation of cyclic-adenosine monophosphate (cAMP) (Fig. 4a). The fusion of BeWo cells by forskolin is confirmed by increasing hCG concentrations in the culture supernatant (Fig. 4b). The expression of senescence-related proteins in groups treated with and without forskolin is investigated using Western blots. The present results indicate that the expression of hCG and p21 is stronger in forskolin-treated cells 24,48 , and $72 \mathrm{~h}$ after its addition. On the other hand, the expression of PML is slightly weaker in forskolin-treated cells (Fig. 4b). The complete loss of p16 expression is observed in BeWo cells. These results indicate that BeWo cells are senescent in association with cell fusion, and accurately reflect senescence in vivo.

\subsection{Graphic summary of the senescence and proliferation of trophoblast}

CT proliferate in the first and second trimesters, and simultaneously 


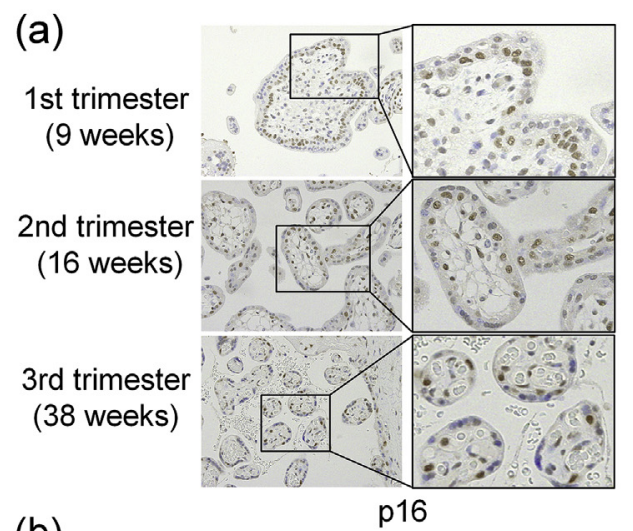

(b)

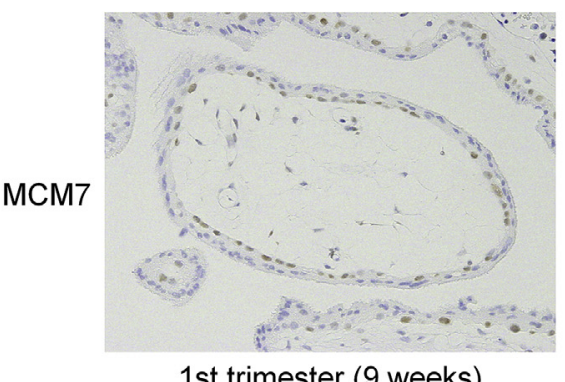

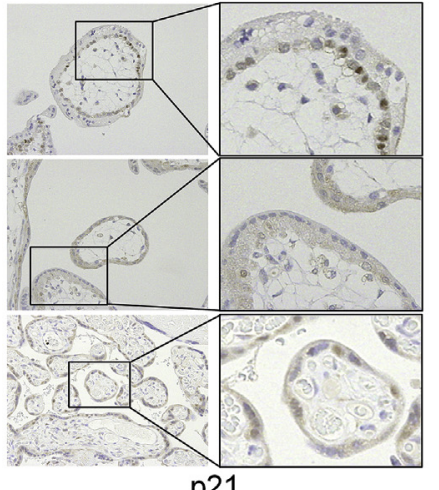

p21

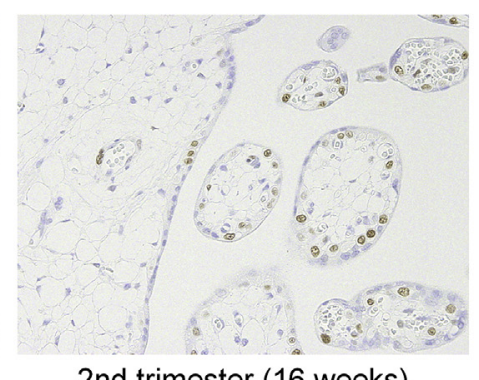

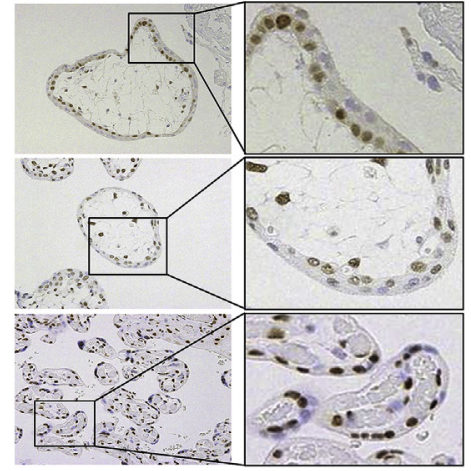

PML

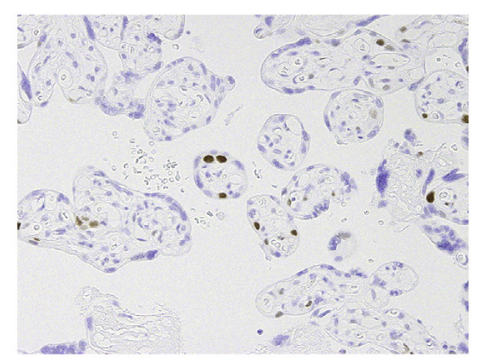

3rd trimester (38 weeks)

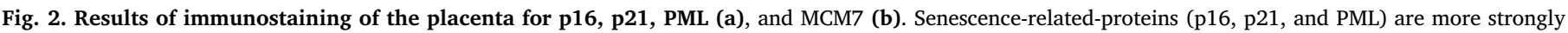

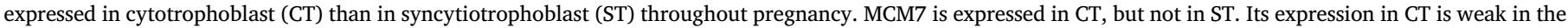
third trimester.
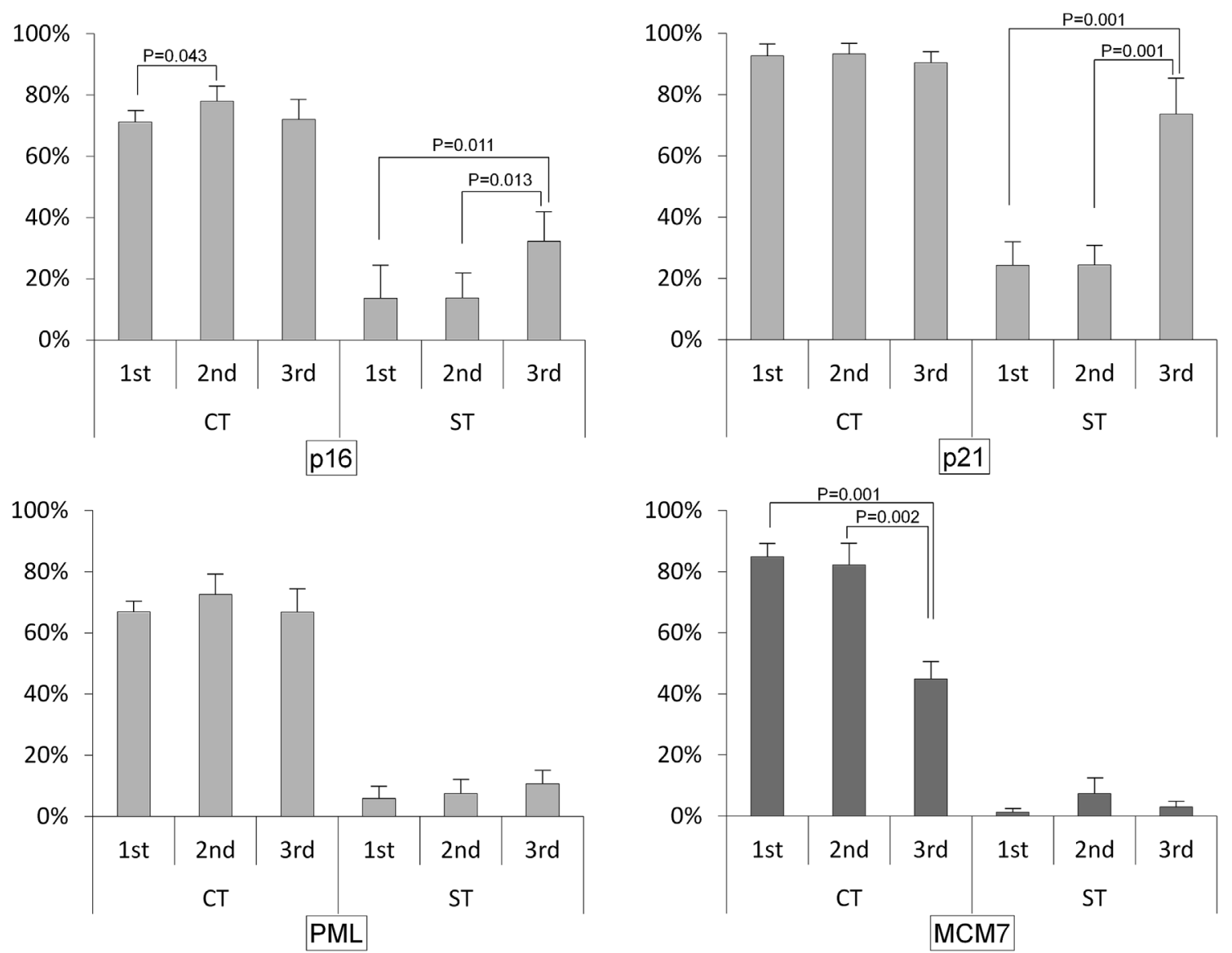

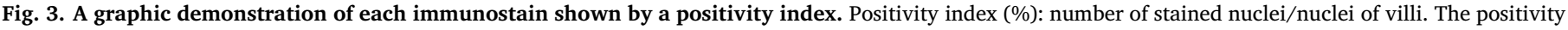
index of cytotrophoblast (CT) is high in all trimesters, but significantly increases in syncytiotrophoblast (ST) as pregnancy advances. 
(a)

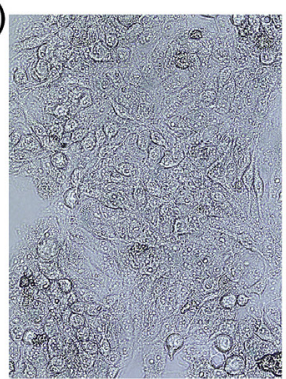

$24 \mathrm{~h}$ forskolin (-)

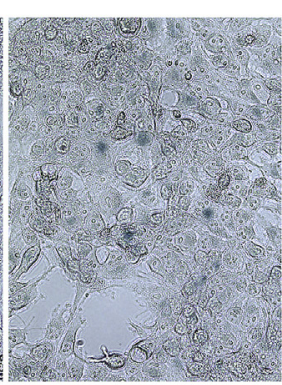

$24 \mathrm{~h}$ forskolin (+)

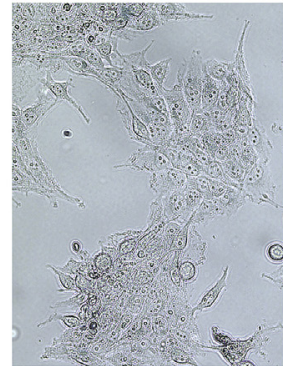

$48 \mathrm{~h}$ forskolin (-)

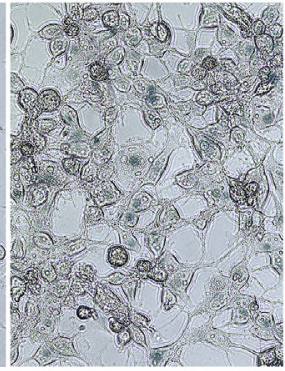

$48 \mathrm{~h}$ forskolin (+)
Fig. 4. (a) Results of SA- $\beta$-gal staining in BeWo cells. SA- $\beta$-gal-positive cell numbers increase in the forskolin-treated group. (b) Senescence-related protein (p21 and PML) expression in BeWo cells. The concentration of hCG and Western blotting of BeWo cells treated with or without forskolin. The expression of hCG and p21 is stronger in cells treated with forskolin. The expression of PML is slightly weak in the forskolin-treated group.

(b)
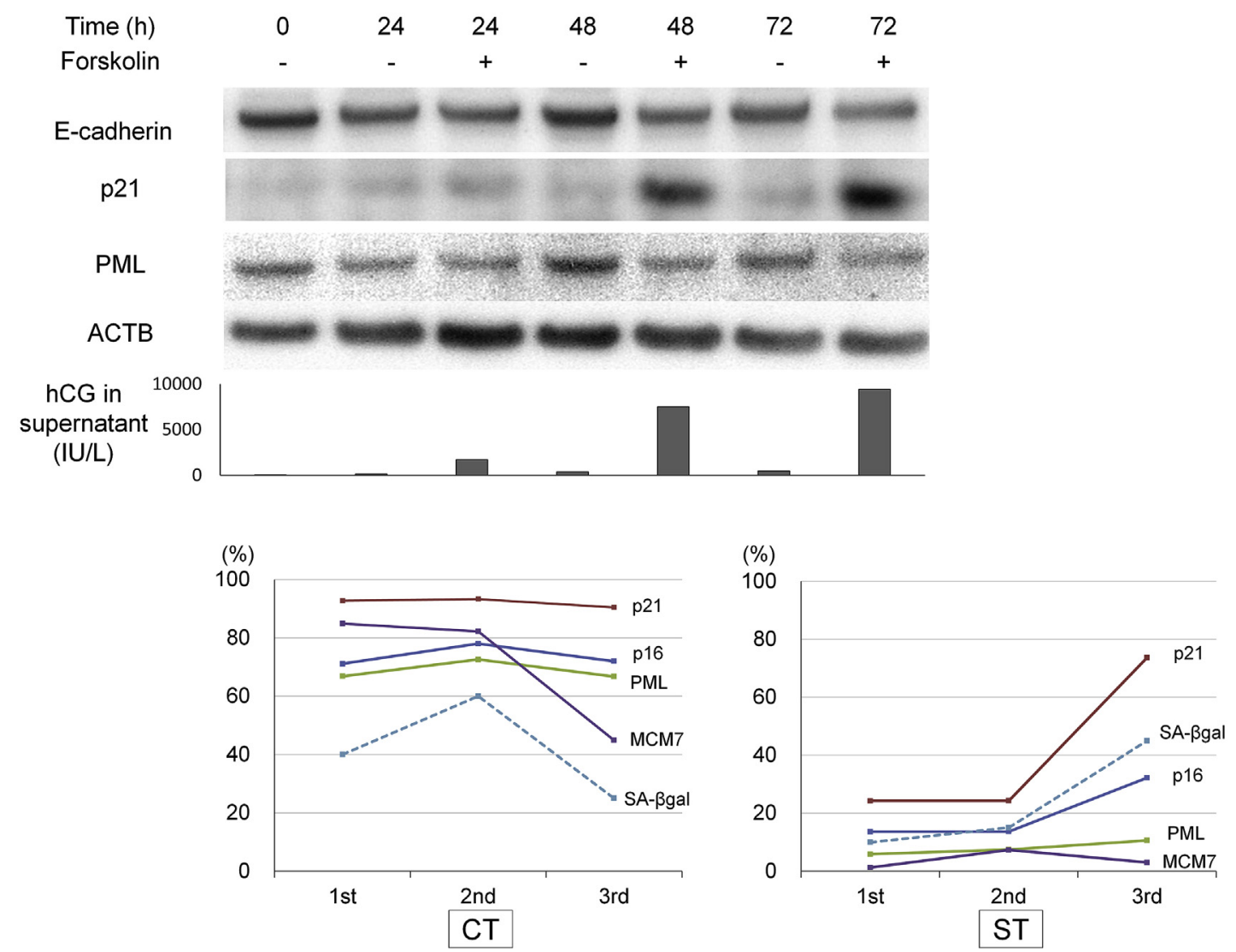

Fig. 5. Graphic summary of results obtained. CT and ST have different senescence/proliferation patterns.

begin to fuse into ST (Fig. 5). Thus, the cell fusion process is considered to indicate the initiation of senescence. ST do not proliferate throughout pregnancy and begin senescence in the third trimester. This cell type-specific proliferation and senescence are considered to be essential for the maintenance of placental function.

\section{Discussion}

The present study demonstrated that the senescence of trophoblastic cells had two peaks during pregnancy: the first peak in CT in the first and second trimesters, and the second peak in ST in the third trimester. We also demonstrated that the forskolin-induced fusion of BeWo cells, an in vitro model of CT, induced senescence, which mirrored senescence during the syncytial fusion of CT into ST in vivo. Cell type-specific senescence appears to be important for the maintenance of placental function.

Cellular senescence is characterized by irreversible growth arrest and altered function [17]. Senescent cells are characterized by their inability to undergo DNA synthesis [17]. Several biomarkers are currently used to detect senescence. Of these, the method to detect SA- $\beta$ - gal using the artificial substrate X-gal developed by Dimri is a convenient, single cell-based assay that identifies senescent cells even in heterogeneous cell populations and aging tissues [18].

P16 is an inhibitor of a cyclin-dependent kinase that directly binds to CDK4 and CDK6, thereby blocking the phosphorylation of the retinoblastoma tumor suppressor (RB) and subsequent progression of the G1 to S phase of the cell cycle [19]. This blockade by the chronic expression of p16 eventually leads to irreversible cell-cycle arrest, termed "cellular senescence" [19]. p21 is another important CDK inhibitor [19]. DNA damage or other stressors activate the tumor suppressor p53, leading to the transient expression of the cyclin-dependent kinase inhibitor (CKI) p21. This triggers momentary G1 cell cycle arrest or leads to a chronic state of senescence or apoptosis.

PML, a key modulator of tumor suppressive pathways, including the p53 and pRb pathways [20], is an essential component of nuclear structures known as PML oncogenic domains (PODs) or nuclear bodies (NBs) [20,21]. PML is known to regulate apoptosis, growth suppression, and senescence. PML has been shown to co-localize with more than 30 different proteins, and direct interactions have been reported for $\mathrm{p} 53$, pRb, DAXX, and CBP [20,22]. Importantly, the overexpression of PML 
in primary fibroblasts is sufficient to cause senescence in a similar manner to RasV12 [23]. PML expression has also been associated with the activation of the $\mathrm{p} 16 / \mathrm{pRb}$ pathway, suggesting that PML coordinately modulates the p53 and pRb master regulator pathways of senescence [24]. In the present study, the pregnancy-related expression of these senescence markers correlated with each other.

Senescence was initially observed in CT, particularly in the first and second trimesters, in the present study. The mRNA expression data of CT and ST in the first trimester reported by Okae et al. also indicated that p16 mRNA expression was detected in CT, but was almost completely lost in ST [25]. It is important to note that cell proliferation, detected by MCM7, was also activated in CT. These results suggest that CT actively proliferated in the early stages of pregnancy, and then promptly proceeded to senescence when the syncytial fusion of CT occurred. Syncytial fusion is regarded as a functional back up of ST by CT [10], as a reservoir of ST. Therefore, CT falls into senescence in this status as the longevity of CT ends, suggesting a change in the role of CT from proliferation to senescence.

In BeWo cells, forskolin-induced cell fusion resulted in the expression of SA- $\beta$-gal and p21. A similar finding was reported in human fetal lung IMR-90 cells; cell fusion caused by the measles virus resulted in the stronger expression of senescence markers, including SA- $\beta$-gal and p21, in fused multi-nucleated cells than in mononuclear cells [12]. Lu et al. reported that $\mathrm{p} 21$ cooperates with the transcription factor GCM1 to induce Syncytin-2 expression in CT, thereby causing cell fusion [26], suggesting that p21 plays an important role in the cell fusion of CT. However, mononuclear CT already expressed elevated levels of senescence markers in early pregnancy, the reason for which currently remains unclear. Huang et al. reported that the mouse embryonic endoderm just after implantation was positive for SA- $\beta$-gal and p21, but was not in an irreversible senescent status, indicating that the expression of SA- $\beta$-gal and p21 was positive in actively proliferating cells [27]. Thus, the elevated expression of SA- $\beta$-gal and p21 implies the proliferative activity of CT, which form ST in later pregnancy. It was not possible to investigate the expression of p16 in association with cell fusion using BeWo cells because of the loss of p16 expression, which is frequently observed in many cancer cells [28]. Collectively, these results indicate that this two-sided function, proliferation-associated senescence is a hallmark of CT. In the first half of pregnancy, CT vigorously proliferate. Several growth factors for CT have been reported to date [29]. However, once these growth factors are depleted, cells appear to promptly become senescent. Kent et al. showed that the PI3K pathway was involved in trophoblast cell differentiation [30]. MTOR (mechanistic target of rapamycin kinase) is a well-known key molecule of the Phosphoinositide 3-kinase (PI3K)-mTOR pathway contributing to cell proliferation as well as a critical factor involved in the induction of cellular senescence. Previous studies demonstrated that rapamycin, an mTOR inhibitor, prevented cellular senescence [31,32]. There is currently no information on the factors influencing the fate of CT.

Another important result of the present study is the senescence of ST in the third trimester. This result was plausible because the main component of the placental villi in the third trimester is ST [33], and ST often exhibit morphological changes such as cell flattening with pyknotic nuclei, suggesting the aging of cells [34]. Although SA- $\beta$-gal activity is a sensitive biochemical marker, only a limited number of studies have examined this activity in the human placenta. Chuprin et al. reported the strong expression of SA- $\beta$-gal in multinuclear ST in the third trimester placenta after the onset of labor, whereas that in CT was negative [12]. However, they did not examine the third trimester placenta before the onset of delivery or senescence in the first and second trimesters. Biron-Shental et al. showed that the percentages of SA- $\beta$-gal in the third trimester placentas of normal and diabetic pregnancies were 0.8 and $7.1 \%$, respectively [35]. However, they did not distinguish ST from CT. In the present study, we demonstrated that the expression of SA- $\beta$-gal in ST was negligible in the first and second trimesters, but increased in the third trimester. Chuprin et al. was the first to report the elevated expression of the senescence markers p21 and p16 in ST in the third trimester [12]. Zhang et al. showed the expression of p21 and p53 in the mouse trophoblastic layer in late pregnancy [36]. These findings are consistent with the present results. The present results also revealed the elevated expression of another senescence marker, PML, in third trimester ST. Collectively, these results showed increased senescence in ST in the third trimester, suggesting the involvement of replicative senescence [37]. Telomere length is reportedly linked to replicative senescence [37]. Gielen et al. found that telomere length linearly decreased by $25 \%$ between 28 and 42 weeks of pregnancy [38]. Therefore, further studies are needed to measure telomere lengths in CT and ST during pregnancy.

Previous studies reported a relationship between the onset of labor and inflammatory cytokines. More recently, the relationship between inflammatory cytokines and senescent cells has been attracting increasing attention; cytokines, such as IL- 6 and IL-8, are reportedly secreted from senescent cells and form a non-bacterial inflammatory milieu, termed SASP [39]. Velarde et al. found that fetal membrane cells exhibited a senescent nature and also that SASP-related cytokine levels increased in amniotic fluid after the onset of labor, indicating that the senescence of the fetal membrane is involved in the onset of labor [40]. Menon et al. reported that senescence was induced in fetal membrane cells by telomere fragments, and this resulted in the onset of labor [41]. However, the present study demonstrated that ST become senescent in the third trimester, which potentially induced the onset of labor.

In conclusion, the present results indicated that the expression patterns of SA- $\beta$-gal and senescence-associated proteins differed between CT and ST. Furthermore, using an in vitro model of BeWo cells, we demonstrated that the process of syncytial fusion was associated with increased senescence, reflecting the phenomenon in vivo. To the best of our knowledge, this is the first study to show two peaks of senescence in CT and ST. This cell type-specific, coordinated appearance of senescence is pivotal to the maintenance of placental function and, potentially, the induction of labor.

\section{Declarations of interest}

The authors declare that there are no conflicts of interest.

\section{Acknowledgments}

This work was supported by Japan Society for the Promotion of Science, Japan; Grant-in-Aid for Scientific Research (KAKENHI), Grant Number 15K15601.

\section{Appendix A. Supplementary data}

Supplementary data to this article can be found online at https:// doi.org/10.1016/j.placenta.2019.06.377.

\section{References}

[1] J.A. Keelan, Intrauterine inflammatory activation, functional progesterone withdrawal, and the timing of term and preterm birth, J. Reprod. Immunol. 125 (2018) 88-99 https://doi.org/10.1016/j.jri.2017.12.004.

[2] S.H. Kim, P.R. Bennett, V. Terzidou, Advances in the role of oxytocin receptors in human parturition, Mol. Cell. Endocrinol. 449 (2017) 56-63 https://doi.org/10. 1016/j.mce.2017.01.034.

[3] R. Smith, R.C. Nicholson, Corticotrophin releasing hormone and the timing of birth, Front. Biosci. 12 (2007) 912-918.

[4] J.M. Bowen, L. Chamley, J.A. Keelan, M.D. Mitchell, Cytokines of the placenta and extra-placental membranes: roles and regulation during human pregnancy and parturition, Placenta 23 (2002) 257-273 https://doi.org/10.1053/plac.2001.0782.

[5] H.C. Nadeau, A. Subramaniam, W.W. Andrews, Infection and preterm birth, Semin Fetal Neonatal Med. 21 (2016) 100-105 https://doi.org/10.1016/j.siny.2015.12. 008 .

[6] D. Stygar, H. Wang, Y.S. Vladic, G. Ekman, H. Eriksson, L. Sahlin, Increased level of matrix metalloproteinases 2 and 9 in the ripening process of the human cervix, Biol. 
Reprod. 67 (2002) 889-894.

[7] N.S. Green, K. Damus, J.L. Simpson, J. Iams, E.A. Reece, C.J. Hobel, I.R. Merkatz, M.F. Greene, R.H. Schwarz, Research agenda for preterm birth: recommendations from the March of Dimes, Am. J. Obstet. Gynecol. 193 (2005) 626-635 https://doi. org/10.1016/j.ajog.2005.02.106.

[8] C.D. Wiley, J. Campisi, From ancient pathways to aging cells-connecting metabolism and cellular senescence, Cell Metabol. 23 (2016) 1013-1021 https://doi.org/ 10.1016/j.cmet.2016.05.010.

[9] J.P. Coppé, P.Y. Desprez, A. Krtolica, J. Campisi, The senescence-associated secretory phenotype: the dark side of tumor suppression, Annu. Rev. Pathol. 5 (2010) 99-118 https://doi.org/10.1146/annurev-pathol-121808-102144.

[10] M. Gauster, B. Huppertz, Fusion of cytotrophoblast with syncytiotrophoblast in the human placenta: factors involved in syncytialization, J. Reprod. Med. Endocrinol. 5 (2008) 76-82.

[11] P. Gerbaud, G. Pidoux, Review: an overview of molecular events occurring in human trophoblast fusion, Placenta 36 (2015) 35-42 https://doi.org/10.1016/j. placenta.2014.12.015.

[12] A. Chuprin, H. Gal, T. Biron-Shental, A. Biran, A. Amiel, S. Rozenblatt, V. Krizhanovsky, Cell fusion induced by ERVWE1 or measles virus causes cellular senescence, Genes Dev. 27 (2013) 2356-2366 https://doi.org/10.1101/gad. 227512.113

[13] T.M. Mayhew, Turnover of human villous trophoblast in normal pregnancy: what do we know and what do we need to know? Placenta 35 (2014) 229-240 https:// doi.org/10.1016/j.placenta.2014.01.011.

[14] S.J. Holt, Indigogenic staining methods for esterases, in: J.F. Danielli (Ed.), General Cytochemical Methods, vol. 1, Academic Press Inc., New York, 1958, pp. 375-398.

[15] H. Kobara, T. Miyamoto, A. Suzuki, R. Asaka, Y. Yamada, K. Ishikawa, N. Kikuchi, S. Ohira, T. Shiozawa, Lipocalin2 enhances the matrix metalloproteinase- 9 activity and invasion of extravillous trophoblasts under hypoxia, Placenta 34 (2013) 1036-1043 https://doi.org/10.1016/j.placenta.2013.08.004.

[16] D. Hinode, H. Hayashi, R. Nakamura, Purification and characterization of three types of proteases from culture supernatants of Porphyromonas gingivalis, Infect. Immun. 59 (1991) 3060-3068.

[17] J. Campisi, F. d'Adda di Fagagna, Cellular senescence: when bad things happen to good cells, Nat. Rev. Mol. Cell Biol. 8 (2007) 729-740 https://doi.org/10.1038/ nrm2233.

[18] K. Itahana, J. Campisi, G.P. Dimri, Methods to detect biomarkers of cellular senescence: the senescence-associated beta-galactosidase assay, Methods Mol. Biol. 371 (2007) 21-31.

[19] J.M. van Deursen, The role of senescent cells in ageing, Nature 509 (2014) 439-446 https://doi.org/10.1038/nature13193.

[20] K.S. Hsu, H.Y. Kao, PML: regulation and multifaceted function beyond tumor suppression, Cell Biosci. 8 (2018) 5 https://doi.org/10.1186/s13578-018-0204-8.

[21] V. Lallemand-Breitenbach, $\mathrm{H}$. de Thé, PML nuclear bodies, Cold Spring Harb. Perspect Biol. 2 (2010) a000661 https://doi.org/10.1101/cshperspect.a000661.

[22] P. Salomoni, The PML-interacting protein DAXX: histone loading gets into the picture, Front Oncol. 152 (2013) 152 https://doi.org/10.3389/fonc.2013.00152.

[23] M. Pearson, R. Carbone, C. Sebastiani, M. Cioce, M. Fagioli, S. Saito, Y. Higashimoto, E. Appella, S. Minucci, P.P. Pandolfi, P.G. Pelicci, PML regulates p53 acetylation and premature senescence induced by oncogenic Ras, Nature 406 (2000) 207-210 https://doi.org/10.1038/35018127.

[24] G. Schmid, M.P. Kramer, M. Maurer, S. Wandl, J. Wesierska-Gadek, Cellular and organismal ageing: role of the p53 tumor suppressor protein in the induction of transient and terminal senescence, J. Cell. Biochem. 101 (2007) 355-369 https:// doi.org/10.1002/jcb.21383.

[25] H. Okae, H. Toh, T. Sato, H. Hiura, S. Takahashi, K. Shirane, Y. Kabayama, M. Suyama, H. Sasaki, T. Arima, Derivation of human trophoblast stem cells, Cell Stem Cell 22 (2018) 50-63.

[26] X. Lu, R. Wang, C. Zhu, H. Wang, H.Y. Lin, Y. Gu, J.C. Cross, H. Wang, Fine-tuned and cell-cycle-restricted expression of fusogenic protein syncytin-2 maintains functional placental syncytia, Cell Rep. 21 (2017) 1150-1159.

[27] T. Huang, J.A. Rivera-Pérez, Senescence-associated $\beta$-galactosidase activity marks the visceral endoderm of mouse embryos but is not indicative of senescence Genesis 52 (2014) 300-308 https://doi.org/10.1002/dvg.22761.

[28] A. Kubo, K. Nakagawa, R.K. Varma, N.K. Conrad, J.Q. Cheng, W.C. Lee, J.R. Testa, B.E. Johnson, F.J. Kaye, M.J. Kelley, The p16 status of tumor cell lines identifies small molecule inhibitors specific for cyclin-dependent kinase 4, Clin. Cancer Res. 12 (1999) 4279-4286.

[29] S.K. Gupta, S.S. Malhotra, A. Malik, S. Verma, P. Chaudhary, Cell signaling path ways involved during invasion and syncytialization of trophoblast cells, Am. J. Reprod. Immunol. 75 (2016) 361-371 https://doi.org/10.1111/aji.12436.

[30] L.N. Kent, T. Konno, M.J. Soares, Phosphatidylinositol 3 kinase modulation of trophoblast cell differentiation, BMC Dev. Biol. 10 (2010) 97, https://doi.org/10. 1186/1471-213X-10-97.

[31] Z.N. Demidenko, S.G. Zubova, E.I. Bukreeva, V.A. Pospelov, T.V. Pospelova, M.V. Blagosklonny, Rapamycin decelerates cellular senescence, Cell Cycle 8 (2009) 1888-1895.

[32] Z.N. Demidenko, M.V. Blagosklonny, Growth stimulation leads to cellular senescence when the cell cycle is blocked, Cell Cycle 7 (2008) 3355-3361.

[33] T.M. Mayhew, Turnover of human villous trophoblast in normal pregnancy: what do we know and what do we need to know? Placenta 35 (2014) 229-240 https:// doi.org/10.1016/j.placenta.2014.01.011.

[34] D. Goldman-Wohl, S. Yagel, United we stand not dividing: the syncytiotrophoblast and cell senescence, Placenta 35 (2014) 341-344 https://doi.org/10.1016/j placenta.2014.03.012.

[35] T. Biron-Shental, R. Sukenik-Halevy, H. Naboani, M. Liberman, R. Kats, A. Amiel, Telomeres are shorter in placentas from pregnancies with uncontrolled diabetes, Placenta 36 (2015) 199-203 https://doi.org/10.1016/j.placenta.2014.11.011.

[36] K. Zhang, C. Chen, Y. Liu, H. Chen, J.P. Liu, Cellular senescence occurred widespread to multiple selective sites in the fetal tissues and organs of mice, Clin. Exp. Pharmacol. Physiol. 41 (2014) 965-975 https://doi.org/10.1111/1440-1681. 12328

[37] C.P. Chiu, C.B. Harley, Replicative senescence and cell immortality: the role of telomeres and telomerase, Proc. Soc. Exp. Biol. Med. 214 (1997) 99-106.

[38] M. Gielen, G. Hageman, D. Pachen, C. Derom, R. Vlietinck, M.P. Zeegers, Placental telomere length decreases with gestational age and is influenced by parity: a study of third trimester live-born twins, Placenta 35 (2014) 791-796 https://doi.org/10. 1016/j.placenta.2014.05.010.

[39] F. Rodier, J. Campisi, Four faces of cellular senescence, J. Cell Biol. 192 (2011) 547-556 https://doi.org/10.1083/jcb.201009094.

[40] R. Menon, F. Behnia, J. Polettini, G.R. Saade, J. Campisi, M. Velarde, Placental membrane aging and HMGB1 signaling associated with human parturition, Aging (Albany NY) 8 (2016) 216-230 https://doi.org/10.18632/aging.100891.

[41] J. Polettini, F. Behnia, B.D. Taylor, G.R. Saade, R.N. Taylor, R. Menon, Telomere fragment induced amnion cell senescence: a contributor to parturition? PLoS One 10 (2015) e0137188https://doi.org/10.1371/journal.pone.0137188. 